\title{
KummerDirichlet Distributions of Matrix Variate in the Complex Case
}

\author{
${ }^{1}$ Ms. SamtaGulia, ${ }^{2}$ Prof. (Dr.) Harish Singh \\ ${ }^{1}$ Research Scholar, NIMS University, Shobha Nagar, Jaipur-Delhi Highway (NH-11C),Jaipur- \\ 303121,Rajasthan, INDIA. \\ ${ }^{2}$ Professor, Department of Business Administration,Maharaja Surajmal Institute(Affiliated to Guru Gobind \\ Singh Indraprastha University),C-4,Janak Puri, New Delhi-110058, INDIA.
}

Abstract: The aim of this paper is to investigate matrix variate generalizations of multivariate Kummer-Beta and multivariate Kummer-Gamma families of distributions in the complex case. The multivatiateKummer-Beta and multivariate Kummer-Gamma families of distributions have been proposed and studied recently by $\mathrm{Ng}$ and Kotz. These distributions are extensions of Kummer-Beta and Kummer-Gamma distributions. Many known or new results have been made with the help of multivatiateKummer-Beta and multivariate Kummer-Gamma families of distributions.

\section{Introduction}

The Kummer-Beta and kummer-Gamma families of distributions are defined by the density functions involving hermition positive definite matrix

$$
\frac{\Gamma(\alpha+\beta)}{\Gamma(\alpha) \Gamma(\beta)}\left\{{ }_{1} \mathrm{~F}_{1}(\alpha ; \alpha+\beta ;-\lambda)\right\}^{-1} \operatorname{ex}(-\lambda \mathrm{u}) \mathrm{u}^{\alpha-1}(1-\mathrm{u}) \beta-1,0<\mathrm{u}<1,
$$

$\{\Gamma(\alpha) \psi(\alpha, \alpha-\gamma+; \varepsilon)\}^{-1} \exp (-\varepsilon v) v^{\alpha-1}(1+v)^{-\gamma}, v>0$,

respectively, where $\alpha>0, \beta>0, \varepsilon>0,-\infty<\gamma, \lambda<\infty,{ }_{1} \mathrm{~F}_{1}$, and $\psi$ are confluent hypergeometric functions. These distributions are extensions of Gamma and Beta distributions, and for $\alpha<1$ (and certain values of $\lambda$ and $\gamma$ ) yield bimodal distributions on finite and infinite ranges, respectively. These distributions are used (i) in the 3 Bayesian analysis of queueing system where posterior distribution of certain basic parameters in M / M / oqueueing system is Kummer-Gamma and (ii) in common value auctions where the posterior distribution of "value of a single good" is Kummer-Beta. For properties and applications of these distributions the reader is referred to NG and Kotz [7], Armero and Bayarri [1], and Gordy [2].

As the corresponding multivariate generalization of these distributions, we have the following $n$ dimensional densities:

$$
\begin{aligned}
& \frac{\tilde{\Gamma}\left(\sum_{i=1}^{n} \alpha_{i}+\beta\right)}{\prod_{i=1}^{n} \tilde{\Gamma}\left(\alpha_{i}\right) \tilde{\Gamma}(\beta)}\left\{{ }_{1} \mathrm{~F}_{1}\left(\sum_{i=1}^{n} \alpha_{i} ; \sum_{i=1}^{n} \alpha_{i}+\beta ;-\lambda\right)\right\}^{-1} \exp \left(-\lambda \sum_{i=1}^{n} u_{i}\right) \\
& \times \prod_{i=1}^{n} u_{i}^{a_{i}-1}\left(1-\sum_{i=1}^{n} u_{i}\right)^{\beta-1}, 0<u_{i}<1, \sum_{i=1}^{n} u_{i}<1,
\end{aligned}
$$

Where $\alpha_{i}>0, i=1, \ldots ., n, \beta>0,-\infty<\lambda<\infty$, and

$$
\begin{aligned}
& \left\{\widetilde{\Gamma}\left(\sum_{i=1}^{n} \alpha_{i}\right) \psi\left(\sum_{i=1}^{n} \alpha_{i}, \sum_{i=1}^{n} \alpha_{i}-\lambda+1 ; \varepsilon\right)\right\}^{-1} \exp \left(-\varepsilon \sum_{i=1}^{n} v_{i}\right) \\
& \times \prod_{i=1}^{n} v_{i}^{a_{i}-1}\left(1+\sum_{i=1}^{n} v_{i}\right)^{-\gamma}, v_{i}>0,
\end{aligned}
$$

Where $\alpha_{i}>0, i=1, \ldots \ldots ., n, \varepsilon>0,-\infty<\gamma<\infty$, respectively. These distributions have been considered by $\mathrm{Ng}$ and Kotz [7] who refer to (1.3) and (1.4) as multivariate Kummer-Beta and multivariate Kummer-Gamma distributions, respectively. For $\lambda=0,(1.1)$ and (1.3) reduce to Beta and Dirichlet distributions with probability density functions

$$
\frac{\tilde{\Gamma}(\alpha+\beta)}{\tilde{\Gamma}(\alpha) \tilde{\Gamma}(\beta)} u^{\alpha-1}(1-u)^{\beta-1}, 0<u<1,
$$




$$
\frac{\tilde{\Gamma}\left(\sum_{i=1}^{n} \alpha_{i}+\beta\right)}{\prod_{i=1}^{n} \tilde{\Gamma}\left(\alpha_{i}\right) \tilde{\Gamma}(\beta)} \prod_{i=1}^{n} u_{i}^{a_{i}-1}\left(1-\sum_{i=1}^{n} u_{i}\right)^{\beta-1}, 0<u_{i}<1, \sum_{i=1}^{n} u_{i}<1
$$

respectively. Since (1.3) is an extension of Dirichlet distribution and a multivariate generalization of Kummer-Beta distribution, anppropriate nomenclature for this distribution would be Kummer-Dirichlet distribution. In the same vein, we may call (1.4) a Kummer-Dirichlet distribution. Further, in order to distinguish between these two distributions ((1.3) and (1.4)), we call them Kummer-Dirichlet type I and Kummer-Dirichlet type II distributions.

In this article we propose and study matrix variate generalizations of (1.3) and (1.4), respectively.

\section{Matrix variateKummer-Dirichlet distributions in the complex case}

We begin with a brief review of some definitions and notations. We adhere to standard notations (cf. Gupta and Nagar [3]). Let $A=\left(a_{i j}\right)$ be a $\mathrm{p} \times \mathrm{p}$ matrix.

Then, $\mathrm{A}^{\prime}$ denotes the transpose of $\mathrm{A} ; \operatorname{tr}(\mathrm{A})=\mathrm{a}_{11} \ldots+\mathrm{a}_{\mathrm{pp}}$; $\operatorname{etr}(\mathrm{A})=\exp (\operatorname{tr}(\mathrm{A}))$;

$\operatorname{det}(\mathrm{A})=\operatorname{determinant}$ of $\quad \mathrm{A} ; \mathrm{A}>0$ means that $\mathrm{A}$ is hermition square root of $\mathrm{A}>0$.

The multivariate gamma function $\tilde{\Gamma}_{\mathrm{p}}(\mathrm{m})$ is defined as

$$
\tilde{\Gamma}_{\mathrm{p}}(m)=\pi^{\mathrm{p}(\mathrm{p}-1) / 4} \prod_{j=1}^{p} \tilde{\Gamma}\left(m-\frac{j-1}{2}\right), \operatorname{Re}(m)>(\mathrm{p}-1)
$$

where $\operatorname{Re}($.$) denotes the real part of (.). It is straightforward to show that$

$$
\tilde{\Gamma}_{\mathrm{p}}(m)=\int_{\mathrm{R}>0} \operatorname{det}(\overline{\mathrm{R}})^{m-p} \operatorname{etr}(\overline{\mathrm{R}}) \mathrm{d} \overline{\mathrm{R}}, \operatorname{Re}(m)>(\mathrm{p}-1)
$$

Where the integral has been evaluated over the space of the $p \times$ phermition positive definite matrices. The integral representation of the confluent hypergeometric function ${ }_{1} \mathrm{~F}_{1}$ is given by $1 \mathrm{~F} 1(\mathrm{a} ; \mathrm{b} ; \overline{\mathrm{X}})=\frac{\widetilde{\Gamma}_{p}(b)}{\widetilde{\Gamma}_{p}(a) \widetilde{\Gamma}_{p}(b-a)} \times \int_{<\mathrm{R}<\mathrm{I}_{\mathrm{p}}} \operatorname{det}(\overline{\mathrm{R}})^{a-p} \operatorname{det}\left(\mathrm{I}_{\mathrm{p}}-\overline{\mathrm{R}}\right)^{b-a-p} \operatorname{etr}(\mathrm{X} \overline{\mathrm{R}}) \mathrm{d} \overline{\mathrm{R}}$,

where $\operatorname{Re}(a)>(\mathrm{p}-1)$ and $\operatorname{Re}(\mathrm{b}-\mathrm{a})>(\mathrm{p}-1)$. The confluent hypergeometric function $\psi$ of a $\mathrm{p} \times \mathrm{p}$ hermition matrix $\mathrm{X}$ is defined by

$$
\psi(\mathrm{a}, \mathrm{c} ; \mathrm{X})=\frac{1}{\widetilde{\Gamma}_{p}(a)} \times \int_{\mathrm{R}>0} \operatorname{etr}(-\mathrm{X} \overline{\mathrm{R}}) \operatorname{det}(\overline{\mathrm{R}})^{a-p} \operatorname{det}\left(\mathrm{I}_{\mathrm{p}}+\overline{\mathrm{R}}\right)^{\mathrm{c}-\mathrm{a}-\mathrm{p}} \overline{\mathrm{R}},
$$

where $\operatorname{Re}(\overline{\mathrm{X}})>0$ and $\operatorname{Re}(a)>(p-1)$

Now we define the corresponding matrix variate generalizations of (1.3) and (1.4) as follows

Definition 2.1 The $p \times p$ Hermition positive definite random matrices $\mathrm{u}_{1}, \ldots, \mathrm{u}_{n}$ are said to have the matrix variate Kummer-Drichlet type I distribution with parameters $\alpha_{1}, \ldots, \alpha_{n}, \beta$ and $\Lambda$, denoted by $\left(\mathrm{u}_{1}, \ldots, \mathrm{u}_{n}\right) \sim \mathrm{KD}_{\mathrm{p}}^{\mathrm{I}}\left(\alpha_{1}, \ldots, \alpha_{n}, \beta, \Lambda\right)$, if their joint probability density function (pdf) is given by

$$
\begin{aligned}
\mathrm{K}_{1}\left(\alpha_{1}, \ldots, \alpha_{n}, \beta, \Lambda\right) \operatorname{etr}\left(-\Lambda \sum_{i=1}^{n} \overline{\mathrm{u}}_{i}\right) \\
\times \prod_{i=1}^{n} \operatorname{det}\left(\overline{\mathrm{u}}_{i}\right)^{\alpha_{i}-p} \operatorname{det}\left(\mathrm{I}_{\mathrm{p}}-\sum_{i=1}^{n} \overline{\mathrm{u}}_{i}\right)^{\beta-p} \\
0<\overline{\mathrm{u}}_{i}<\mathrm{I}_{\mathrm{p}}, 0<\sum_{i=1}^{n} \overline{\mathrm{u}}_{i}<\mathrm{I}_{\mathrm{p}}
\end{aligned}
$$

where $\alpha_{i}>(\mathrm{p}-1) i=1, \ldots, \mathrm{n}, \beta>(\mathrm{p}-1) \Lambda(\mathrm{p} \times \mathrm{p})$ is Hermition $\mathrm{K}_{1}\left(\alpha_{1}, \ldots, \alpha_{n}, \beta, \Lambda\right)$ is the normalizing constant.

Definition 2.2. The $\mathrm{p} \times \mathrm{pHermiotion}$ positive definite random matrices $\mathrm{V}_{1}, \ldots, \mathrm{V}_{n}$ are said to have the matrix variateKummer-Dirichlet type II distribution with parameters $\alpha_{1}, \ldots ., \alpha_{n}, \gamma$ and $\Xi$, denoted by $\left(\mathrm{V}_{1}, \ldots, \mathrm{V}_{n}\right) \sim \mathrm{KD}_{\mathrm{p}}^{\mathrm{II}}\left(\alpha_{1}, \ldots, \alpha_{n}, \gamma, \Xi\right)$, if their joint pdf is given by 


$$
\begin{aligned}
\mathrm{K}_{2}\left(\alpha_{1}, \ldots, \alpha_{n}, \gamma, \Xi\right) \operatorname{etr}\left(-\Xi \sum_{i=1}^{n} \overline{\mathrm{V}}_{i}\right) \\
\quad \times \prod_{i=1}^{n} \operatorname{det}\left(\overline{\mathrm{V}}_{i}\right)^{\alpha_{i}-p} \operatorname{det}\left(\mathrm{I}_{\mathrm{p}}-\sum_{i=1}^{n} \overline{\mathrm{V}}_{i}\right)^{-\gamma}, \mathrm{V} i>0,
\end{aligned}
$$

where $\alpha_{i}>(\mathrm{p}-1), i=1, \ldots, \mathrm{n},-\infty<\gamma<\infty, \Xi(\mathrm{p} \times \mathrm{p})>0$, and $\mathrm{k}_{2}\left(\alpha_{1}, \ldots, \alpha_{n}, \gamma, \Xi\right)$ IS The normalizing constant.

The normalizing constants in (2.5) and (2.6) are given as

$$
\begin{aligned}
& \left\{\mathrm{K}_{1}\left(\alpha_{1}, \ldots, \alpha_{n}, \beta, \Lambda\right)\right\}^{-1} \\
& =\int_{\substack{0<\sum_{i=1}^{n} \\
u_{i}>0}} \ldots \int_{u_{i}<\mathrm{I}_{\mathrm{P}}} \operatorname{etr}\left(-\Lambda \sum_{i=1}^{n} \overline{\mathrm{u}}_{i}\right) \\
& \times \prod_{i=1}^{n} \operatorname{det}\left(\overline{\mathrm{u}}_{i}\right)^{\alpha_{i}-p} \operatorname{det}\left(\mathrm{I}_{\mathrm{p}}-\sum_{i=1}^{n} \overline{\mathrm{u}}_{i}\right)^{\beta-p} \prod_{i=1}^{n} \mathrm{~d} \overline{\mathrm{u}}_{i} \\
& =\frac{\prod_{i=1}^{n} \tilde{\Gamma}_{p}\left(\alpha_{i}\right)}{\tilde{\Gamma}_{p}\left(\sum_{i=1}^{n} \alpha_{i}\right)} \int_{0<u<\mathrm{I}_{\mathrm{p}}} \operatorname{etr}(-\Lambda \overline{\mathrm{u}}) \operatorname{det}(\overline{\mathrm{u}}) \sum_{i=1}^{n} \alpha_{i}-p \\
& x \operatorname{det}\left(\mathrm{I}_{\mathrm{p}}-\overline{\mathrm{u}}\right)^{\beta-\mathrm{p}} \mathrm{d} \overline{\mathrm{u}} \\
& =\frac{\prod_{i=1}^{n} \tilde{\Gamma}_{p}\left(\alpha_{i}\right) \tilde{\Gamma}_{p}\left(\beta_{i}\right)}{\tilde{\Gamma}_{p}\left(\sum_{i=1}^{n} \alpha_{i}+\beta\right)} 1 \mathrm{~F} 1\left(\sum_{i=1}^{n} \alpha_{i} ; \sum_{i=1}^{n} \alpha_{i}+\beta ;-\Lambda\right), \\
& \left\{\mathrm{K}_{2}\left(\alpha_{1}, \ldots, \alpha_{n}, \gamma, \Xi\right)\right\}^{-1} \\
& =\int_{V_{1}>0} \ldots \int_{V_{1}>0} \operatorname{etr}\left(-\Xi \sum_{i=1}^{n} \overline{\mathrm{V}}_{i}\right) \\
& \times \prod_{i=1}^{n} \operatorname{det}\left(\overline{\mathrm{V}}_{i}\right)^{\alpha_{i}-p} \operatorname{det}\left(\mathrm{I}_{\mathrm{p}}-\sum_{i=1}^{n} \overline{\mathrm{V}}_{i}\right)^{-\gamma} \prod_{i=1}^{n} \mathrm{~d} \overline{\mathrm{V}}_{i} \\
& =\frac{\prod_{i=1}^{n} \tilde{\Gamma}_{p}\left(\alpha_{i}\right)}{\tilde{\Gamma}_{p}\left(\sum_{i=1}^{n} \alpha_{i}\right)} \int_{V>0} \operatorname{etr}(-\Xi \overline{\mathrm{V}}) \operatorname{det}(\overline{\mathrm{V}}) \sum_{i=1}^{n} \alpha_{i}-p \operatorname{det}\left(\mathrm{I}_{\mathrm{p}}+\overline{\mathrm{V}}\right)^{-\gamma} \mathrm{d} \overline{\mathrm{V}} \\
& =\prod_{i=1}^{n} \tilde{\Gamma}_{p}\left(\alpha_{i}\right) \psi\left(\sum_{i=1}^{n} \alpha_{i} ; \sum_{i=1}^{n} \alpha_{i}+\gamma+p ; \Xi\right) \text {, }
\end{aligned}
$$

respectively, where ${ }_{1} \mathrm{~F}_{1}$ and $\psi$ are confluent hypergeometric functions of matrix argument.

For $\Lambda=0$, the matrix variateKummer-Dirichlet type I distribution collapses to an ordinary matrix variateDirichlet type I distribution with pdf

$$
\begin{gathered}
\frac{\tilde{\Gamma}_{p}\left(\sum_{i=1}^{n} \alpha_{i}+\beta\right)}{\prod_{i=1}^{n} \tilde{\Gamma}_{p}\left(\alpha_{i}\right) \tilde{\Gamma}_{p}\left(\beta_{i}\right)} \prod_{i=1}^{n} \operatorname{det}\left(\overline{\mathrm{u}}_{\mathrm{i}}\right)^{\alpha i-\mathrm{p}} \operatorname{det}\left(\mathrm{I}_{\mathrm{p}}-\sum_{i=1}^{n} \overline{\mathrm{u}}_{i}\right)^{\beta-p} \\
0<\overline{\mathrm{u}}_{\mathrm{i}}<\mathrm{I}_{\mathrm{p}}, 0<\sum_{i=1}^{n} \overline{\mathrm{u}}_{i}<\mathrm{I}_{\mathrm{p}}
\end{gathered}
$$

where $\alpha_{\mathrm{i}}>(\mathrm{p}-1) \mathrm{i}=1, \ldots ., \mathrm{n}$, and $\beta>(\mathrm{p}-1) / 2$. A common notation to designate that $\left(\mathrm{u}_{1}, \ldots, \mathrm{u}_{n}\right)$ has this density is $\left(\mathrm{u}_{1}, \ldots \mathrm{u}_{\mathrm{n}}\right) \sim \mathrm{D}_{\mathrm{P}}^{\mathrm{I}}\left(\alpha_{1}, \ldots, \alpha_{n} ; \beta\right)$. For $\gamma=0$, the matrix variateKummer-Dirichlet type II density simplifies to the product of $\mathrm{n}$ matrix variate Gamma densities.

For $\mathrm{p}=1$, the densities in (2.5) and (2.6) simplify to Kummer-Dirichlet type I (multivariate Kummer Beta) and Kummer - Dirichlet type II distributions reduce to the matrix variateKummer-Beta and matrix variateKummer-Gamma distributions, respectively. These two distributions have been studied by Nagar and Gupta [6] and Nagar and Cardeno [5]. Substituting $n=1$ in (2.5) and (2.6), the matrix variateKummer-Beta and matrix variateKummer-Gamma densities are obtained as 
$\mathrm{K}_{1}(\alpha, \beta, \Lambda) \operatorname{etr}(-\Lambda \overline{\mathrm{u}}) \operatorname{det}(\overline{\mathrm{u}})^{\alpha-\mathrm{P}}$

$\times \operatorname{det}\left(\mathrm{I}_{\mathrm{p}}-\overline{\mathrm{u}}\right)^{\beta-\mathrm{p}}, 0<\overline{\mathrm{u}}<\mathrm{I}_{\mathrm{p}}$,

$\mathrm{K}_{2}(\alpha, \gamma, \Xi) \operatorname{etr}(-\Xi \overline{\mathrm{V}}) \operatorname{det}(\overline{\mathrm{V}})^{\alpha-p} \operatorname{det}\left(\mathrm{I}_{\mathrm{p}}+\overline{\mathrm{V}}\right)^{-\gamma}, \mathrm{V}>0$

respectively, where $\alpha>(\mathrm{p}-1), \beta>(\mathrm{p}-1),-\infty, \Lambda=\Lambda^{\prime}$, and $\Xi(\mathrm{p} \times \mathrm{p})>0$. These two distributions are designated by $\mathrm{u} \sim \mathrm{KB}_{\mathrm{p}}(\alpha, \beta, \Lambda)$ and $\mathrm{V} \sim \mathrm{KG}_{\mathrm{p}}(\alpha, \gamma, \Xi)$. It may be noted that the matrix variateKummerDirichlet distributions are special cases of the matrix variateLiouville distribution.

Using certain transformations, generalized matrix variateKummer-Dirichlet distributions are generated as given in the next two theorems.

Theorem 1. Let $\left(\bar{u}_{1}, \ldots, \bar{u}_{n}\right) \sim \operatorname{KD}_{\mathrm{p}}^{\mathrm{I}}\left(\alpha_{1}, \ldots, \alpha_{n}, \beta, \Lambda\right)$ and $\psi_{1}, \ldots, \psi_{\mathrm{n}}, \Omega$ be hermitionmatricies such that $\Omega>0$ and $\Omega-\sum_{i=1}^{n} \quad \psi_{\mathrm{i}}>0$. Define

$$
\mathrm{Zi}=\left(\Omega-\sum_{i=1}^{n} \psi_{i}\right)^{1 / 2} \mathrm{u}_{i}\left(\Omega-\sum_{i=1}^{n} \psi_{i}\right)^{1 / 2}+\psi_{i}, i=1, \ldots, \mathrm{n} .
$$

The $\left(\mathrm{Z}_{1}, \ldots, \mathrm{Z}_{n}\right)$ have the generalized matrix variateKummer-Dirichlet type I distribution with pdf

$$
\begin{gathered}
\frac{K_{1}\left(\alpha_{1}, \ldots, \alpha_{n}, \beta, \Lambda\right)}{\operatorname{etr}\left(\Omega-\sum_{i=1}^{n} \psi_{i}\right)^{\sum_{i=1}^{n} \alpha_{i}+\beta-p_{i}}} \\
\times \frac{\prod_{i=1}^{n} \operatorname{det}\left(\overline{\mathrm{Z}}_{i}-\psi_{i}\right)^{\alpha_{i}-p} \operatorname{det}\left(\Omega-\sum_{i=1}^{n} \overline{\mathrm{Z}}_{i}\right)^{\beta-p}}{\operatorname{etr}\left\{\left(\Omega-\sum_{i=1}^{n} \psi_{i}\right)^{-1 / 2} \Lambda\left(\Omega-\sum_{i=1}^{n} \psi_{i}\right)^{-1 / 2} \sum_{i=1}^{n}\left(\mathrm{Z}_{i}-\psi_{i}\right)\right\}} \\
\psi_{i}<\overline{\mathrm{Z}}_{i}<\Omega, i=1, \ldots, \mathrm{n}, \sum_{i=1}^{n} \overline{\mathrm{Z}}_{i}<\Omega .
\end{gathered}
$$

Proof. Making the transformation $\overline{\mathrm{u}}_{i}=\left(\Omega-\sum_{i=1}^{n} \psi_{i}\right)^{-1 / 2}\left(\mathrm{Z}_{i}-\psi_{i}\right)\left(\Omega-\sum_{i=1}^{n} \psi_{i}\right)^{-1 / 2}, i=1, \ldots, n$, with Jacobin $\mathrm{J}\left(\mathrm{u}_{1}, \ldots, \mathrm{u}_{n} \rightarrow \mathrm{Z}_{1}, \ldots, \mathrm{Z}_{n}\right)=\operatorname{det}\left(\Omega-\sum_{i=1}^{n} \psi_{i}\right)^{-\mathrm{np}}$ in $(2.5)$, we $\operatorname{get}(2.12)$.

If $\left(\mathrm{Z}_{1}, \ldots, \mathrm{Z}_{n}\right)$ has the $\operatorname{pdf}(2.12)$, then we write $\left(\mathrm{Z}_{1}, \ldots, \mathrm{Z}_{n}\right) \sim \mathrm{GK} \mathrm{D}_{\mathrm{P}}^{\mathrm{I}}\left(\alpha_{1}, \ldots, \alpha_{n}, \beta, \Lambda ; \Omega ; \psi_{1}, \ldots, \psi_{\mathrm{n}}\right)$. Note that $\operatorname{GK}_{\mathrm{P}}^{\mathrm{I}}\left(\alpha_{1}, \ldots, \alpha_{n}, \beta, \Lambda ; \mathrm{I}_{\mathrm{p}} ; 0, \ldots, 0\right) \equiv \mathrm{KD}_{\mathrm{P}}^{\mathrm{I}}\left(\alpha_{1}, \ldots, \alpha_{n}, \beta, \Lambda\right)$.

Theorem 2. Let $(\mathrm{V} 1, \ldots, \mathrm{Vn}) \sim \mathrm{KD}_{\mathrm{P}}^{\mathrm{II}}\left(\alpha_{1}, \ldots, \alpha_{n}, \gamma, \Xi\right)$ and $\psi_{1}, \ldots, \psi_{\mathrm{n}}, \Omega$ be hermition matrices such that $\Omega>0$ and $\Omega+\sum_{i=1}^{n} \psi_{1}>0$. Define

$$
Y_{\mathrm{i}}=\left(\Omega-\sum_{i=1}^{n} \psi_{i}\right)^{1 / 2} \mathrm{~V}_{i}\left(\Omega-\sum_{i=1}^{n} \psi_{i}\right)^{1 / 2}+\psi_{i}, i=1, \ldots, \mathrm{n} .
$$

Then, $\left(Y_{1}, \ldots, Y_{n}\right)$ have the generalized matrix variaeKummer-Dirichlet type II distribution with pdf

$$
\begin{aligned}
& \frac{K_{2}\left(\alpha_{1}, \ldots, \alpha_{n}, \gamma, \Xi\right)}{\operatorname{det}\left(\Omega-\sum_{i=1}^{n} \psi_{i}\right)^{\sum_{i=1}^{n} \alpha_{i}-\gamma}} \\
& \times \frac{\prod_{i=1}^{n} \operatorname{det}\left(\overline{\mathrm{Y}}_{i}-\psi_{i}\right)^{\alpha_{i}-p} \operatorname{det}\left(\Omega-\sum_{i=1}^{n} \overline{\mathrm{Y}}_{i}\right)^{-\gamma}}{\operatorname{etr}\left\{\left(\Omega-\sum_{i=1}^{n} \psi_{i}\right)^{-1 / 2} \Xi\left(\Omega-\sum_{i=1}^{n} \psi_{i}\right)^{-1 / 2} \sum_{i=1}^{n}\left(\mathrm{Y}_{i}-\psi_{i}\right)\right\}} \\
& \qquad \mathrm{Y}_{i}>\psi_{i}, \mathrm{i}=1, \ldots, \mathrm{n} .
\end{aligned}
$$

Proof. Making the transformation $\left.\mathrm{V}_{i}=\left(\Omega+\sum_{i=1}^{n} \psi_{i}\right)^{-1 / 2}\right)\left(\mathrm{Y}_{i}-\psi_{i}\right)\left(\Omega+\sum_{i=1}^{n} \psi_{i}\right)^{-1 / 2}, i=1, \ldots, \mathrm{n}$, with the Jacobian $\mathrm{J}\left(\mathrm{V}_{1}, \ldots, \mathrm{V}_{\mathrm{n}} \rightarrow \mathrm{Y}_{1}, \ldots, \mathrm{Y}_{\mathrm{n}}\right)=\operatorname{det}\left(\Omega+\sum_{i=1}^{n} \psi_{i}\right)^{-n p}$ in (2.6), we get (2.14).

If $\left(\mathrm{Y}_{1}, \ldots, \mathrm{Y}_{\mathrm{n}}\right)$ has pdf $(2.14)$, then we write $\left(\mathrm{Y}_{1}, \ldots, \mathrm{Y}_{\mathrm{n}}\right) \sim \mathrm{GK}_{\mathrm{P}}^{\mathrm{II}}\left(\alpha_{1}, \ldots, \alpha_{n}, \gamma, \Xi ; \Omega \psi_{1}, \ldots, \psi_{\mathrm{n}}\right)$. In this case GK $\mathrm{D}_{\mathrm{P}}^{\mathrm{II}}\left(\alpha_{1}, \ldots, \alpha_{n}, \gamma, \mathrm{I}_{\mathrm{p}} ; 0, \ldots, 0\right) \equiv \mathrm{KD}_{\mathrm{P}}^{\mathrm{II}}\left(\alpha_{1}, \ldots, \alpha_{n} ; \gamma, \Xi\right)$.

\section{Properties}


In this section, we study certain properties of matrix variateKummer-Dirichlet type I and II invariant. That is, for any fixed orthogonal matrix $\Gamma(\mathrm{p} \times \mathrm{p})$, the distribution of $\left(\Gamma \mathrm{u}_{1} \Gamma^{\prime}, \ldots, \Gamma \mathrm{u}_{\mathrm{n}} \Gamma^{\prime}\right)$ is the same as the distribution of $\left(\mathrm{u}_{1}, \ldots, \mathrm{u}_{\mathrm{n}}\right)$. Our next two results give marginal and conditional distributions.distributions. It may be noted that for $\Lambda=\lambda \mathrm{I}_{\mathrm{p}}, \Xi=\varepsilon \mathrm{I}_{\mathrm{p}}$ densities (2.5) and (2.6) are othogonaily

Theorem 3. If $\left(\mathrm{u}_{1}, \ldots, \mathrm{u}_{\mathrm{n}}\right) \sim \mathrm{KD}_{\mathrm{P}}^{\mathrm{I}}\left(\alpha_{1}, \ldots, \alpha_{n}, \beta, \Lambda\right)$, then the joint marginal pdf of $\mathrm{u}_{1}, \ldots, \mathrm{u}_{\mathrm{m}}, \mathrm{m} \leq \mathrm{n}$, is given by

$$
\begin{aligned}
\mathrm{K}_{1}\left(\alpha_{1}, \ldots, \alpha_{m}, \sum_{i=m+1}^{n} \alpha_{i}+\beta, \Lambda\right) \operatorname{etr}\left(-\Lambda \sum_{i=1}^{m} \overline{\mathrm{u}}_{i}\right) \\
\times \prod_{i=1}^{m} \operatorname{det}\left(\overline{\mathrm{u}}_{i}\right)^{\alpha_{i}-p} \operatorname{det}\left(\mathrm{I}_{\mathrm{p}}-\sum_{i=1}^{m} \overline{\mathrm{u}}_{i}\right)^{\sum_{i=m+1}^{n} \alpha_{i}+\beta-p} \\
\times 1 \mathrm{~F} 1\left(\sum_{i=m+1}^{n} \alpha_{i} ; \sum_{i=m+1}^{n} \alpha_{i}+\beta ;-\Lambda\left(\mathrm{I}_{\mathrm{p}}-\sum_{i=1}^{m} \overline{\mathrm{u}}_{i}\right)\right) \\
0<\overline{\mathrm{u}}_{i}<\mathrm{I}_{\mathrm{p}}, 0<\sum_{i=1}^{m} \overline{\mathrm{u}}_{i}<\mathrm{I}_{\mathrm{p}},
\end{aligned}
$$

and the conditional density of $\left(\mathrm{u}_{\mathrm{m}}+1, \ldots, \mathrm{u}_{\mathrm{n}}\right) \mid\left(\mathrm{u}_{1}, \ldots, \mathrm{u}_{\mathrm{m}}\right)$ is given by

$$
\begin{aligned}
& \frac{K_{1}\left(\alpha_{1}, \ldots, a_{n}, \beta, \Lambda\right)}{K_{1}\left(\alpha_{1}, \ldots, a_{m}, \sum_{i=m+1}^{n} \alpha_{i}+\beta, \Lambda\right)} . \\
& \times \frac{\operatorname{etr}\left(-\Lambda \sum_{i=m+1}^{n} \overline{\mathrm{u}}_{i}\right)}{\operatorname{det}\left(\mathrm{I}_{\mathrm{p}}-\sum_{i=1}^{m} \overline{\mathrm{u}}_{i}\right)^{\sum_{i=m+1}^{n} \alpha_{i}+\beta-p}} \\
& \times \frac{\prod_{i=m+1}^{n} \operatorname{det}\left(\overline{\mathrm{u}}_{i}\right)^{\alpha_{i}-p} \operatorname{det}\left(\mathrm{I}_{\mathrm{p}}-\sum_{i=1}^{m} \overline{\mathrm{u}}_{i}-\sum_{i=m+1}^{n} \overline{\mathrm{u}}_{i}\right)^{\beta-p}}{{ }_{1} \mathrm{~F}_{1}\left(\sum_{i=m+1}^{n} \alpha_{i} ; \sum_{i=m+1}^{n} \alpha_{i}+\beta ;-\Lambda\left(\mathrm{I}_{\mathrm{p}} \sum_{i=m+1}^{n} \overline{\mathrm{u}}_{i}\right)\right)} \\
& \quad 0<\overline{\mathrm{u}}_{i} \mathrm{Ip}-\sum_{i=1}^{m} \bar{u}_{i}, \mathrm{i}=m+1, \ldots, n, \sum_{i=m+1}^{m} \bar{u}_{i}<\mathrm{I}_{\mathrm{p}}-\sum_{i=1}^{m} \bar{u}_{i}
\end{aligned}
$$

Proof. First we find the marginal density of $\mathrm{u}_{1}, \ldots, \mathrm{u}_{n-1}$ by integrating out un from the joint density of $\mathrm{u}_{1}, \ldots, \mathrm{u}_{\mathrm{n}}$ as

$$
\begin{aligned}
\mathrm{K}_{1}\left(\alpha_{1}, \ldots, \alpha_{\mathrm{n}}, \beta, \Lambda\right) \int_{0<u_{n}<I_{p}-\sum_{i=1}^{n-1}} \operatorname{etr}\left(-\sum_{i=1}^{m} \bar{u}_{i}\right) \\
\times \prod_{i=1}^{n} \operatorname{det}\left(\overline{\mathrm{u}}_{i}\right)^{\alpha_{i}-p} \operatorname{det}\left(\mathrm{I}_{\mathrm{p}}-\sum_{i=1}^{m} \overline{\mathrm{u}}_{i}\right)^{\beta-p} \mathrm{~d} \overline{\mathrm{u}}_{n} .
\end{aligned}
$$

Now, substituting $\mathrm{Zn}=\left(\mathrm{I}_{\mathrm{p}^{-}} \sum_{i=1}^{n-1} \overline{\mathrm{u}}_{i}\right)^{-1 / 2} \mathrm{u}_{\mathrm{n}}\left(\mathrm{I}_{\mathrm{p}}-\sum_{i=1}^{n-1} \overline{\mathrm{u}}_{i}\right)^{-1 / 2}$ with Jacobian $\mathrm{J}\left(\overline{\mathrm{u}}_{n} \rightarrow \mathrm{Z}_{\mathrm{n}}\right)=\operatorname{det}\left(\mathrm{I}_{\mathrm{p}}-\right.$ $\left.\sum_{i=1}^{n-1} \overline{\mathrm{u}}_{i}\right)^{\mathrm{p}}$ in (3.2), we get

$$
\begin{aligned}
\mathrm{K}_{1}\left(\alpha_{1}, \ldots, \alpha_{\mathrm{n}}, \beta, \Lambda\right) \operatorname{etr}\left(-\Lambda \sum_{i=1}^{n-1} \overline{\mathrm{u}}_{i}\right) \\
\times \prod_{i=1}^{n-1} \operatorname{det}\left(\overline{\mathrm{u}}_{i}\right)^{\alpha_{i}-p} \operatorname{det}\left(\mathrm{I}_{\mathrm{p}}-\sum_{i=1}^{n-1} \overline{\mathrm{u}}_{i}\right)^{\alpha_{n}+\beta-p} \\
\times \int_{0<\mathrm{Z}_{\mathrm{n}}<\mathrm{I}_{\mathrm{p}}} \operatorname{etr}\left[-\left(-\mathrm{I}_{\mathrm{p}}-\sum_{i=1}^{n-1} \overline{\mathrm{u}}_{i}\right)^{1 / 2} \Lambda\left(-\mathrm{I}_{\mathrm{p}}-\sum_{i=1}^{n-1} \overline{\mathrm{u}}_{i}\right)^{1 / 2} \overline{\mathrm{Z}}_{n}\right]
\end{aligned}
$$




$$
\times \operatorname{det}\left(\overline{\mathrm{Z}}_{n}\right)^{\alpha_{n}-p} \operatorname{det}\left(\mathrm{I}_{\mathrm{p}}-\overline{\mathrm{Z}}_{n}\right)^{\beta-\mathrm{p}} \mathrm{d} \overline{\mathrm{Z}}_{n} .
$$

But

$$
\begin{aligned}
& \mathrm{K}_{1}\left(\alpha_{1}, \ldots, \alpha_{\mathrm{n}}, \beta, \Lambda\right) \\
& \quad \times \int_{0<\mathrm{Z}_{\mathrm{r}}<\mathrm{I}_{\mathrm{p}}} \operatorname{etr}\left[-\left(-\mathrm{I}_{\mathrm{p}}-\sum_{i=1}^{n-1} \overline{\mathrm{u}}_{i}\right)^{1 / 2} \Lambda\left(-\mathrm{I}_{\mathrm{p}}-\sum_{i=1}^{n-1} \overline{\mathrm{u}}_{i}\right)^{1 / 2} \overline{\mathrm{Z}}_{n}\right] \\
& \quad \times \operatorname{det}\left(\overline{\mathrm{Z}}_{n}\right)^{\alpha_{n}-p} \operatorname{det}\left(\mathrm{I}_{\mathrm{p}}-\overline{\mathrm{Z}}_{n}\right)^{\beta-\mathrm{p}} \mathrm{d} \overline{\mathrm{Z}}_{n} . \\
& \mathrm{K}_{1}\left(\alpha_{1}, \ldots, \alpha_{\mathrm{n}}, \beta, \Lambda\right) \frac{\tilde{\Gamma}_{p}\left(\alpha_{n}\right) \tilde{\Gamma}_{p}(\beta)}{\widetilde{\Gamma}_{p}\left(\alpha_{n}+\beta\right)}{ }_{1} \mathrm{~F}_{1}\left(\alpha_{n} ; \alpha_{n}+\beta ;-\Lambda\left(\mathrm{I}_{\mathrm{p}}-\sum_{i=1}^{n-1} \overline{\mathrm{u}}_{i}\right)\right) \\
& =\mathrm{K}_{1}\left(\alpha_{1}, \ldots, \alpha_{\mathrm{n}},-1, \alpha_{\mathrm{n}}+\beta, \Lambda\right)\left(\alpha_{n} ; \alpha_{n}+\beta ;-\Lambda\left(\mathrm{I}_{\mathrm{p}}-\sum_{i=1}^{n-1} \overline{\mathrm{u}}_{i}\right)\right)
\end{aligned}
$$

Hence, we get the joint density of $\left(\mathrm{u}_{1}, \ldots, \mathrm{u}_{\mathrm{n}-1}\right)$ as

$$
\begin{aligned}
& \mathrm{K}_{1}\left(\alpha_{1}, \ldots, \alpha_{\mathrm{n}},-1, \alpha_{\mathrm{n}}+\beta, \Lambda\right) \operatorname{etr}\left(\mathrm{I}_{\mathrm{p}}-\sum_{i=1}^{n-1} \overline{\mathrm{u}}_{i}\right) \prod_{i=1}^{n-1} \operatorname{det}\left(\overline{\mathrm{u}}_{i}\right)^{\alpha_{i}-p} \\
& \times \operatorname{det}\left(\mathrm{I}_{\mathrm{p}}-\sum_{i=1}^{n-1} \overline{\mathrm{u}}_{i}\right)^{\alpha_{n}+\beta-p}{ }_{1} \mathrm{~F}_{1}\left(\alpha_{n} ; \alpha_{n}+\beta ;-\Lambda\left(\mathrm{I}_{\mathrm{p}}-\sum_{i=1}^{n-1} \overline{\mathrm{u}}_{i}\right)\right)
\end{aligned}
$$

Repeating this procedure $\mathrm{n}-\mathrm{m}$ times gives the marginal density of $\left(\mathrm{u}_{1}, \ldots, \mathrm{u}_{\mathrm{m}}\right)$ as

$$
\begin{aligned}
& \mathrm{K} 1\left(\alpha_{1}, \ldots, \alpha_{m} \sum_{i=m+1}^{n} \alpha_{i}+\beta, \Lambda\right) \operatorname{etr}\left(-\Lambda \sum_{i=1}^{n-1} \overline{\mathrm{u}}_{i}\right) \\
& \times \prod_{i=1}^{m} \operatorname{det}\left(\overline{\mathrm{u}}_{i}\right)^{\alpha_{i}-p} \operatorname{det}\left(\mathrm{I}_{\mathrm{p}}-\sum_{i=1}^{n-1} \overline{\mathrm{u}}_{i}\right)^{\sum_{i=m}^{n} \alpha_{n}+\beta-p} \\
& \times{ }_{1} \mathrm{~F}_{1}\left(\sum_{i=m+1}^{n} \alpha_{i} ; \sum_{i=m+1}^{n} \alpha_{i}+\beta ;-\Lambda\left(\mathrm{I}_{\mathrm{p}}-\sum_{i=1}^{m} \overline{\mathrm{u}}_{i}\right)\right) .
\end{aligned}
$$

Now the second part of the theorem follows immediately.

Corollary 3.1 - If $\left(\mathrm{u}_{1}, \ldots \mathrm{u}_{\mathrm{n}}\right) \sim \mathrm{KD}_{\mathrm{p}}^{\mathrm{I}}\left(\alpha_{1}, \ldots \alpha_{n}, \beta, \Lambda\right)$, then the marginal pdf of $\mathrm{u}_{i}, i=1, \ldots, n$ is given by

$$
\begin{aligned}
& \mathrm{K}_{1}\left(\alpha_{i}, \sum_{j=1(\neq i)}^{n} \alpha_{j}+\beta ;-\Lambda\right) \operatorname{etr}\left(-\Lambda \overline{\mathrm{u}}_{i}\right) \operatorname{det}\left(\overline{\mathrm{u}}_{i}\right) \alpha_{i}-p \\
& \times \operatorname{det}\left(\mathrm{I}_{\mathrm{p}}-\overline{\mathrm{u}}_{i}\right)^{\sum_{j=1(\neq i)}^{n} \alpha_{j}+\beta-p} \\
& \times_{1} \mathrm{~F}_{1}\left(\alpha_{i}, \sum_{j=1(\neq i)}^{n} \alpha_{j} ;+\sum_{j=1(\neq i)}^{n} \alpha_{j}+\beta-\Lambda\left(\mathrm{I}_{\mathrm{p}}-\overline{\mathrm{u}}_{i}\right)\right), 0<\overline{\mathrm{u}}_{i}<\mathrm{I}_{\mathrm{p}} .
\end{aligned}
$$

It is interesting to note that the marginal density of $\mathrm{u}_{i}$ does not belong to the Kummer-Beta family and differs by an additional factor containing confluent hypergeometric function ${ }_{1} \mathrm{~F}_{1}$.

In Theorem 4, we give results on marginal and conditional distributions for Kummer-Dirchlet type II distribution. Before doing so, we need to give an integral that will be used in the derivation of marginal distribution. From (2.6) and, (2.8) we have

$$
\begin{gathered}
\int_{x>0} \int_{Y>0} \operatorname{etr}[-\Xi][(\overline{\mathrm{X}}+\overline{\mathrm{Y}})] \operatorname{Det}(\overline{\mathrm{Y}})^{a_{1}-p} \\
\quad \times \operatorname{det}(\overline{\mathrm{X}})^{a_{2}-p} \operatorname{det}\left(\mathrm{I}_{\mathrm{p}}+\overline{\mathrm{X}}+\overline{\mathrm{Y}}\right)^{-\mathrm{b}} \mathrm{d} \overline{\mathrm{X}} \mathrm{d} \overline{\mathrm{Y}} \\
=\tilde{\Gamma}_{p}\left(a_{1}\right) \tilde{\Gamma}_{p}\left(a_{2}\right) \psi\left(a_{1}+a_{2}, a_{1}+a_{2}-\mathrm{b}+\mathrm{p} ; \Xi\right),
\end{gathered}
$$

where $\operatorname{Re}\left(a_{1}\right)>(\mathrm{p}-1), \operatorname{Re}\left(a_{2}\right)>(\mathrm{p}-1)$ and $\operatorname{Re} \Xi>0$. Substituting $\mathrm{W}$, we obtain

$\mathrm{W}=\left(\mathrm{I}_{\mathrm{p}}+\mathrm{X}\right)^{-1 / 2} \mathrm{Y}\left(\mathrm{I}_{\mathrm{p}}+\mathrm{X}\right)-1 / 2$ with the Jacobian $\mathrm{J}(\mathrm{Y} \rightarrow \mathrm{W})=\mathrm{DET}(\mathrm{Ip}+\overline{\mathrm{X}})^{\mathrm{p}}$ in (3.9) and integrating 


$$
\begin{aligned}
& \int_{x>0} \operatorname{etr}(-\Xi \overline{\mathrm{X}}) \operatorname{det}(\overline{\mathrm{X}})^{a_{2}-p} \operatorname{det}(\mathrm{Ip}+\overline{\mathrm{X}})^{a_{1}-b} \\
& \times \psi\left(\mathrm{a}_{1}, \mathrm{a}_{1}-\mathrm{b}+\mathrm{p} ; \Xi\left(\mathrm{I}_{\mathrm{p}}+\overline{\mathrm{X}}\right) \mathrm{d} \overline{\mathrm{X}}\right. \\
& =\Gamma_{\mathrm{p}}\left(a_{2}\right) \psi\left(\mathrm{a}_{1}, \mathrm{a}_{1}-\mathrm{b}+\mathrm{p}\right) .
\end{aligned}
$$

Now we turn to our problem of finding the marginal and conditional distributions.

Theorem 4. If $\left(\mathrm{V}_{1}, \ldots, \mathrm{V}_{\mathrm{n}}\right) \sim \mathrm{K} \mathrm{D}_{\mathrm{p}}^{\mathrm{II}}\left(\alpha_{1}, \ldots, \alpha_{\mathrm{n}}, \gamma, \Xi\right)$, the joint marginal pdf of $\mathrm{V}_{1}, \ldots, \mathrm{V}_{m}, m<n$, is given by

$$
\begin{aligned}
& \tilde{\Gamma}_{p}\left(\sum_{i=m+1}^{n} \alpha_{i}\right) \mathrm{K} 2\left(\alpha_{1} \ldots, \alpha_{m}, \sum_{i=m+1}^{n} \alpha_{i}, \gamma, \Xi\right) \operatorname{etr}\left(-\Xi \sum_{i=1}^{m} \overline{\mathrm{V}}_{i}\right) \\
& \times \prod_{i=1}^{m} \operatorname{det}\left(\overline{\mathrm{V}}_{i}\right)^{\alpha_{i}-p} \operatorname{det}\left(I_{p}+\sum_{i=1}^{n} \overline{\mathrm{V}}_{i}\right)^{-\gamma+\sum_{i=m+1}^{n} \alpha_{i}} \\
& \times \psi\left(\sum_{i=m+1}^{n} \alpha_{i}, \sum_{i=m+1}^{n} \alpha_{i}-\gamma+p ; \Xi\left(\mathrm{I}_{\mathrm{p}}+\sum_{i=1}^{m} \overline{\mathrm{V}}_{j}\right)\right), \\
& \mathrm{V}_{j}>0, \mathrm{j}=1, \ldots, \mathrm{m}
\end{aligned}
$$

and the conditional density of $\left(\mathrm{V}_{\mathrm{m}+1}, \ldots, \mathrm{V}_{\mathrm{n}}\right) \mid\left(\mathrm{V}_{1}, \ldots \mathrm{V}_{\mathrm{m}}\right)$ is given by

$$
\begin{aligned}
& \frac{K_{2}\left(\alpha_{1}, \ldots, \alpha_{n}, \gamma, \Xi\right)}{\tilde{\Gamma}_{p}\left(\sum_{i=m+1}^{n} \alpha_{i}\right) K_{2}\left(\alpha_{1}, \ldots, \alpha_{m}, \sum_{i=m+1}^{n} \alpha_{i}, \gamma, \Xi\right)} \\
& \times \frac{\operatorname{etr}\left(-\Xi \sum_{i=m+1}^{n} \overline{\mathrm{V}}_{i}\right)}{\operatorname{det}\left(\mathrm{I}_{\mathrm{p}}+\sum_{i=1}^{m} \overline{\mathrm{V}}_{i}\right)^{-\gamma \sum_{i=m+1}^{n} \alpha_{i}}} \\
& \times \frac{\prod_{i=m+1}^{n} \operatorname{det}\left(\overline{\mathrm{V}}_{i}\right)^{\alpha_{i}-p} \operatorname{det}\left(\mathrm{I}_{\mathrm{p}}+\sum_{i=1}^{m} \overline{\mathrm{V}}_{i}+\sum_{i=1}^{n} \overline{\mathrm{V}}_{i}\right)^{-\gamma}}{\psi\left(\sum_{i=m+1}^{m} \alpha_{\mathrm{i}}, \sum_{i=m+1}^{n} \alpha_{i}-\gamma+p \Xi\left(\mathrm{I}_{\mathrm{p}}+\sum_{j=1}^{m} \overline{\mathrm{V}}_{j}\right)\right)} \mathrm{s} \\
& \quad \mathrm{Vi}>0, \mathrm{i}=\mathrm{m}+1, \ldots, \mathrm{n} .
\end{aligned}
$$

Proof. In this case, to obtain the marginal density of $\mathrm{V} 1, \ldots ., \mathrm{Vn}-1$, we substitute $\mathrm{Wn}=\left(\mathrm{Ip}+\sum_{i=1}^{n-1} \overline{\mathrm{V}}_{i}\right)^{-1 / 2} \mathrm{~V}_{\mathrm{n}}\left(\mathrm{Ip}+\sum_{i=1}^{n-1} \overline{\mathrm{V}}_{i}\right)^{-1 / 2}$ with the jacobian $\mathrm{J}(\mathrm{Vn} \rightarrow \mathrm{Wn})=\operatorname{det}\left(\mathrm{Ip}+\sum_{i=1}^{n-1} \overline{\mathrm{V}}_{i}\right) \mathrm{p}$.

Thus, the joint density of $\mathrm{V}_{1}, \ldots, \mathrm{V}_{\mathrm{n}-1}$ is obtained as

$$
\begin{aligned}
\mathrm{K} 2\left(\alpha_{1}, \ldots, \alpha_{\mathrm{n}}, \gamma, \Xi\right) \operatorname{etr}\left(-\Xi \sum_{i=1}^{n-1} \overline{\mathrm{V}}_{i}\right) \\
\times \prod_{i=1}^{n-1} \operatorname{det}\left(\overline{\mathrm{V}}_{i}\right)^{\alpha_{i}-p} \operatorname{det}\left(I_{p}+\sum_{i=1}^{n-1} \overline{\mathrm{V}}_{i}\right)^{-\gamma+\alpha_{n}} \\
\times \int_{w_{n}>0} \operatorname{etr}\left[-\left(I_{p}+\sum_{i=1}^{n-1} \overline{\mathrm{V}}_{i}\right)^{1 / 2} \Xi\left(I_{p}+\sum_{i=1}^{n-1} \overline{\mathrm{V}}_{i}\right)^{1 / 2} \overline{\mathrm{W}}_{n}\right] \\
\times \operatorname{det}\left(\overline{\mathrm{W}}_{n}\right)^{a_{n}-p} \operatorname{det}\left(\mathrm{I}_{\mathrm{p}}+\overline{\mathrm{W}}_{n}\right)^{-\gamma} \mathrm{d} \overline{\mathrm{W}}_{n} \\
=\Gamma_{\mathrm{p}}\left(\alpha_{n}\right) \mathrm{K}_{2}\left(\alpha_{1}, \ldots, \alpha_{n}, \gamma, \Xi\right) \operatorname{etr}\left(-\Xi \sum_{i=1}^{n-1} \overline{\mathrm{V}}_{i}\right) \\
\times \prod_{i=1}^{n-1} \operatorname{det}\left(\overline{\mathrm{V}}_{i}\right)^{\alpha_{i}-p} \operatorname{det}\left(I_{p}+\sum_{i=1}^{n-1} \overline{\mathrm{V}}_{i}\right)^{-\gamma+\alpha_{n}} \\
\times \psi\left(\alpha_{n}, \alpha_{n}-\gamma+p ; \Xi\left(I_{p}+\sum_{i=1}^{n-1} \overline{\mathrm{V}}_{i}\right)\right)
\end{aligned}
$$


Further, substituting $\mathrm{W}_{\mathrm{n}-1}=\left(\mathrm{I}_{\mathrm{p}}+\sum_{i=1}^{n-2} \overline{\mathrm{V}}_{i}\right)^{-1 / 2} \mathrm{~V}_{\mathrm{n}-1}\left(\mathrm{I}_{\mathrm{p}}+\sum_{i=1}^{n-2} \overline{\mathrm{V}}_{i}\right)-1 / 2$ with the Jacobian $\mathrm{j}\left(\mathrm{V}_{\mathrm{n}-1} \rightarrow\right.$ $\left.\mathrm{W}_{\mathrm{n}-1}\right)=\operatorname{det}\left(\mathrm{I}_{\mathrm{p}}+\sum_{i=1}^{n-2} \overline{\mathrm{V}}_{i}\right)^{\mathrm{p}}$ in (3.13) and integrating Wn-1 using (3.10), we get the joint marginal density of $\mathrm{V}_{1}, \ldots, \mathrm{V}_{\mathrm{n}-2}$ as

$$
\begin{aligned}
& \tilde{\Gamma}_{p}\left(\alpha_{n}\right) \mathrm{K}_{2}\left(\alpha_{1}, \ldots, \alpha_{n}, \gamma, \Xi\right) \operatorname{etr}\left(-\Xi \sum_{i=1}^{n-2} \overline{\mathrm{V}}_{i}\right) \\
& \times \prod_{i=1}^{n-2} \operatorname{det}\left(\overline{\mathrm{V}}_{i}\right)^{\alpha_{i}-p} \operatorname{det}\left(I_{p}+\sum_{i=1}^{n-2} \overline{\mathrm{V}}_{i}\right)^{-\gamma+\alpha_{n}+\alpha_{n-1}} \\
& \times \int_{w_{n}>0} \operatorname{etr}\left[-\left(I_{p}+\sum_{i=1}^{n-2} \overline{\mathrm{V}}_{i}\right)^{1 / 2} \Xi\left(I_{p}+\sum_{i=1}^{n-2} \overline{\mathrm{V}}_{i}\right)^{1 / 2} \overline{\mathrm{W}}_{n-1}\right] \\
& \times \operatorname{det}\left(\overline{\mathrm{W}}_{n-1}\right)^{a_{n}-p} \operatorname{det}\left(\mathrm{I}_{\mathrm{p}}+\overline{\mathrm{W}}_{n-1}\right)^{-\gamma+\alpha \mathrm{n}} \\
& \times \psi\left(\alpha_{n}, \alpha_{n}-\gamma+p ;\left(I_{p}+\sum_{i=1}^{n-2} \overline{\mathrm{V}}_{i}\right)^{1 / 2}\right. \\
& \left.\times \Xi\left(I_{p}+\sum_{i=1}^{n-2} \overline{\mathrm{V}}_{i}\right)^{1 / 2} W_{n-1}\right) d \bar{W}_{n-1} \\
& =\Gamma_{\mathrm{p}}\left(\alpha_{\mathrm{n}}\right) \Gamma_{\mathrm{p}}\left(\alpha_{\mathrm{n}-1}\right) \mathrm{K}_{2}\left(\alpha_{1}, \ldots, \alpha_{\mathrm{n}}, \gamma, \Xi\right) \operatorname{etr} \\
& \left.\times \prod_{i=1}^{n-2} \operatorname{det}\left(-\overline{\mathrm{V}}_{i}\right)^{\alpha_{i}-p} \operatorname{det}\left(I_{p}+\sum_{i=1}^{n-2} \overline{\mathrm{V}}_{i}\right)^{-\gamma+\alpha_{n}+\alpha_{n-1}}\right) \\
& \times \psi\left(\alpha_{n}, \alpha_{n-1}, \alpha_{n}+\alpha_{n-1}-\gamma+p ;\left(I_{p}+\sum_{i=1}^{n-2} \overline{\mathrm{V}}_{i}\right)\right)
\end{aligned}
$$

Integrating out $\mathrm{V}_{\mathrm{n}-2}, \ldots, \mathrm{V}_{\mathrm{m}+1}$ similarly, we get marginal density of $\mathrm{V}_{1}, \ldots, \mathrm{V}_{\mathrm{m}}$ as

$$
\begin{aligned}
& \left.\times \prod_{i=m+1}^{n} \tilde{\Gamma}_{p}\left(\alpha_{1}\right) \mathrm{K}_{2}\left(\alpha_{1}, \ldots, \alpha_{n}, \gamma, \Xi\right) \operatorname{etr}\left(-\Xi \sum_{i=1}^{n-2} \overline{\mathrm{V}}_{i}\right)\right) \\
& \times \prod_{i=1}^{m} \operatorname{det}\left(\overline{\mathrm{V}}_{i}\right)^{\alpha_{i}-p} \operatorname{det}\left(I_{p}+\sum_{i=1}^{m} \overline{\mathrm{V}}_{i}\right)^{-\gamma+\sum_{i=m+i}^{n}+\alpha_{1}} \\
& \times \psi\left(\alpha_{n}, \alpha_{n-1}, \alpha_{n}+\alpha_{n-1}-\gamma+p ;\left(I_{p}+\sum_{i=1}^{n-2} \overline{\mathrm{V}}_{i}\right)\right) \\
& \times \prod_{i=m+1}^{n} \tilde{\Gamma}_{p}\left(\alpha_{1}\right) \mathrm{K}_{2}\left(\alpha_{1}, \ldots, \alpha_{n}, \gamma, \Xi\right) \\
& =\tilde{\Gamma}_{p}\left(\sum_{i=m+1}^{n} \alpha_{i}\right) \mathrm{K}_{2}\left(\alpha_{1}, \ldots, \alpha_{m}, \sum_{i=m+1}^{n} \alpha_{1}, \gamma, \Xi\right) .
\end{aligned}
$$

The derivation of the conditional density is now straightforward.

Corollary 3.2. If $\left(\mathrm{V}_{1}, \ldots, \mathrm{V}_{\mathrm{n}}\right) \sim \mathrm{K} \mathrm{D}_{\mathrm{p}}^{\mathrm{II}}\left(\alpha_{1}, \ldots, \alpha_{\mathrm{n}}, \gamma, \Xi\right)$, then density of $\mathrm{V}_{i}, i=1, \ldots, \mathrm{n}$ is given by

$$
\begin{aligned}
& \tilde{\Gamma}_{p}\left(\sum_{j=1(\neq i)}^{n} \alpha_{i}\right) \mathrm{K}_{2}\left(\alpha_{1}, \sum_{j=1(\neq i)}^{n} \alpha_{1}, \gamma, \Xi\right) \operatorname{etr}\left(-\Xi \overline{\mathrm{V}}_{i}\right) \\
& \times \operatorname{det}\left(\overline{\mathrm{V}}_{i}\right)^{\alpha_{i}-p} \operatorname{det}\left(\mathrm{I}_{\mathrm{p}}+\overline{\mathrm{V}}_{i}\right)^{-\gamma+\sum_{j=1(\neq i)}^{n} \alpha_{j}}
\end{aligned}
$$




$$
\times \psi\left(\sum_{j=1(\neq i)}^{n} \alpha_{1}, \sum_{j=1(\neq i)}^{n} \alpha_{j}-\gamma+p, \Xi\left(I_{p}+\overline{\mathrm{V}}_{i}\right)\right) \mathrm{V}_{i}>0
$$

Note that the marginal density of $\mathrm{V}_{i}$ differs from the Kummer-Gamma density. It is a pdf with an additional factor containing confluent hypergeometricfunction $\psi$.

\section{References}

[1] C. Armero and M. J.Bayarri, A Bayesian analysis of a queneing system with unlimited service, J. Statist. Plann. Inference 58 (1997), no. 2, 241-261. CMP 1 450015.Zbl 880.62025.

[2] M.Gordy, Computationally convenient distributional assumptions for common-value auctions, Comput. Econom. 12 (1998), $61-78$. Zbl 912.90093.

[3] A. K. Gupta and D. K. Nagar, Matrix Variate Distributions, Chapman \& Hall/ CRC Monographs and Surveys in Pure and Applied Mathematics, vol. 104, Chapaman\& Hall/CRC, Florida, 2000. MR 2001d:62055. Zbl 935.62064.

[4] W.R. Javier and A.K. Gupta, On generalized matric variate beta disgtributions, Statistics 16 (1985), no. 4, 549-557, MR 86m:62023. Zbl 602.62039.

[5] D.K. Nagar and L. Cardeno, Matrix variateKummer - Gamma distribution, Random Oper. Stochastic Equations 9 (2001), no. 3 , 207-218.

[6] D.K. Nagar and A.K. Gupta, Matrix variateKummer-Beta distribution, to appear in J. Austral Math.Sec.

[7] K. W. Ng and S. Kotz, Kumer-Gamma and Kummer-Beta univariate and multivariate distributions, Research report, Department of Statics, The University of Hong Kong, Hong Kong, 1995.

[8] Arjun K. Gupta: Department of Mathematics and Statistics, Bowling Green State University, Bowling Green, OH 43403-0221, USA.

[9] Liliamcardeno and Daya K. Nagar: Departmento de Mathematicas, Universidad de Antioquia, Medellin, A. A. 1226, Colombia 